Data are collected in real time, and all partner notification outcomes are recorded. The platform is extendable to facilitate centralised partner notification within sexual health clinical networks across many different heath care providers.

\section{P111 A USER CENTRED APPROACH TO THE DESIGN OF POINT- OF-CARE AND SELF-TEST MOBILE PHONE DIAGNOSTICS FOR SEXUALLY TRANSMITTED INFECTIONS (STIS)}

\author{
doi:10.1136/sextrans-2012-050601c.111
}

${ }^{1} \mathrm{G}$ Voula, ${ }^{*}{ }^{1} \mathrm{~W}$ Balachandran, ${ }^{2} \mathrm{C}$ Lowndes, ${ }^{2} \mathrm{R}$ Howell-Jones, ${ }^{3} \mathrm{C}$ R Aicken, ${ }^{3} \mathrm{C} H$ Mercer, ${ }^{4} \mathrm{~L}$ Sutcliffe, ${ }^{3} \mathrm{P}$ Sonnenberg, ${ }^{5} \mathrm{M}$ Jackson, ${ }^{4} \mathrm{C}$ S Estcourt, ${ }^{6} \mathrm{~S}$ T Sadiq, ${ }^{1} \mathrm{~K}$ Hone. ${ }^{1}$ Brunel University, Middlesex, UK; ${ }^{2}$ Health Protection Agency; ${ }^{3}$ University College London, London, UK; ${ }^{4}$ Queen Mary University of London; ${ }^{5}$ xscient; ${ }^{6}$ St Georges University of London

Background Control of STIs in vulnerable populations is difficult because of inadequate access to prompt diagnosis and treatment and may be enhanced by better community based STI surveillance. The high rates of mobile and smart phone use in these populations and the development of novel personalised diagnostic technologies which theoretically enable highly accurate self-testing diagnostics for STIs that can be electronically linked to clinical care pathways and STI data capture, may help address these challenges. A major challenge for these systems is the development of clinically relevant and acceptable Human Technology Interfaces (HTIs) for Mobile-Web Applications. Objectives To describe a methodological approach to develop a mobile phone/web-based management system that can link rapid self-test diagnostics to clinical care pathways and community based surveillance.

Methods We adopt a user centred approach to the development of a HTI for self-managing STI diagnosis with initial exploratory pilot studies to gather functional, user and clinical requirements of the HTI. Iterative development of functional prototypes exploring design possibilities and technology features is followed by the formative evaluation of interface design alternatives through qualitative and controlled usability studies with target users and existing surveillance capture mechanisms.

Results A user centred, multi-disciplinary approach allows for successful capture and integration of social science methods to inform functional requirements for an effective and user acceptable mobile and web-based self-managing STI diagnostics and surveillance system.

Discussion Future testing in simple and complex, community based studies are needed to primarily assess safety and then effectiveness for improving access to care, enhanced partner notification and development of community based capture of surveillance data.

\section{P112 TEXTING OR TALKING? WHICH WORKS BETTER TO REDUCE HAZARDOUS DRINKING BY SEXUAL HEALTH CLINIC ATTENDEES?}

doi:10.1136/sextrans-2012-050601c.112

\section{S Baguley.* NHS Grampian}

Background Hazardous drinking is associated with an increased risk of catching an STI, getting pregnant unintentionally, being sexually assaulted and sexually assaulting someone else. An Alcohol Brief Intervention ( $\mathrm{ABI}$ ), a type of motivational interview, has been shown to reduce hazardous drinking in men over the age of 25 . There is an paucity of evidence of the benefit in younger people or in women. Texting has been shown to be useful in supporting smoking cessation, weight reduction and diabetes control. There have been no studies of its potential value in reducing hazardous drinking or its consequences.
Aim To compare the effect of texting vs an ABI on hazardous drinking and associated sexual illhealth amongst attendees at an urban sexual health clinic.

Methods Potential subjects are being recruited from a city sexual health clinic. Initial screening is done using the Alcohol Use Disorder Identification Test - a self-completed questionnaire marked out of 40. Consenting subjects are then randomised to texting (12 texts over 4 months), an ABI or a leaflet and then followed up by phone at 4 months. At follow-up, subjects are rescreened for hazardous drinking and asked about incident STIs, regretted sex, sexual assault, use of emergency contraception and for their evaluation of the intervention method.

Results To end of January 2012, 6279 attendees have been screened of whom 2554 were hazardous drinkers and 799 have consented to take part in the study. (The target sample size is 1090). By the time of the conference, all subjects will have been recruited and most results will be available.

Conclusion This is the first RCT of texting for hazardous drinking and should inform national policy in this area.

\section{P113 PRIMARY HEALTH PROVIDERS' ADHERENCE TO NATIONAL CHLAMYDIA GUIDELINES}

doi:10.1136/sextrans-2012-050601c.113

\section{${ }^{1} \mathrm{~J}$ M Morgan, ${ }^{* 2} \mathrm{~L}$ A Mills, ${ }^{1} \mathrm{~A}$ J Bell. ${ }^{1}$ Waikato Hospital; ${ }^{2}$ University of Otago}

Aim To re-audit district-wide management of genital chlamydia infection following implementation of $\mathrm{NZ}$ national chlamydia guidelines.

Methods All healthcare settings in a single NZ health district that diagnosed 20 or more cases of chlamydia during September 2010-2011 were invited to participate and were asked to complete an audit proforma for 20 consecutive cases. Sexual Health clinic cases were excluded. Collated results were compared to findings of a similar audit of 394 cases diagnosed during 2008, which was undertaken before guideline implementation in 2009.

Results 19 of 22 eligible sites participated, providing data for 424 cases; of these, 302 (71.2\%) were managed in General Practice, with the remainder from a range of settings including Family Planning, student health services, and A\&E. 405 of 424 (95.5\%) cases in 2010-2011 had documented treatment compared to 354 of 394 $(89.8 \%)$ in 2008. Notifying current sexual partners was noted in 314 cases $(74 \%)$, compared to $58.1 \%$ in 2008.126 of 424 (29.7\%) of cases were noted to have informed at least $50 \%$ of their sexual partners compared to $17.8 \%$ in 2008 .

Conclusion Improved case management and partner notification may reflect better documentation than in the initial audit but more appropriate choice of diagnostic sampling, more testing for other STIs and more appropriate dosage and duration of prescribed antibiotics supports that primary care chlamydia case management in this district has improved following local implementation of national guidelines. More work is needed to enhance documented partner management outcomes.

\section{Improving clinical practice and service delivery}

\begin{tabular}{l|l}
\hline P114 IS THERE A ROLE FOR PRIMARY CARE CLINICIANS IN \\
PROVIDING SHARED CARE IN HIV TREATMENT? A \\
SYSTEMATIC LITERATURE REVIEW
\end{tabular}

doi:10.1136/sextrans-2012-050601c.114

${ }^{1}$ W Wong, ${ }^{1} \mathrm{C}$ W Luk, ${ }^{2} \mathrm{M}$ R Kidd. ${ }^{1}$ The University of Hong Kong; ${ }^{2}$ Faculty of Health Sciences, Flinders University

Background Despite HIV being increasingly considered as a chronic illness, there is as yet no consensus about how primary care should 
be integrated with specialty care to provide optimal clinical management for people living with HIV.

Objective To examine the effectiveness of shared care models of HIV between primary care and specialty care and how primary care providers can assist in improving the care of people with HIV.

Methods Three databases, PubMed, Medline and EMBase were searched for relevant terms from studies published in the period from 1996 to 2011. Studies were included that integrated primary care in HIV management and included highly active antiretroviral therapy (HAART) as part of the treatment modality.

Results 11 studies that met the inclusion criteria were included in this review. Primary care was found to be at least as effective in HIV counselling, testing and treatment and, to a lesser degree, prevention, when compared to specialty care alone. Screening for HIV at a primary care level was cost-effective, especially in a high HIV prevalence and high-risk community. There were no significant adverse clinical outcomes reported in a primary care approach. Effectiveness of various interventions using a primary care approach were demonstrated in the review, including HAART adherence programmes, home care, the involvement of peer health workers and perinatal use of HAART.

Conclusions Primary care has an important role in the shared care of the diagnosis and management of people with HIV. Some improvements with current guidelines on the management in primary care of people with HIV in developing countries should be considered.

\section{P115 LEARNING FROM PATIENTS TO REDESIGN AN HIV SERVICE}

doi:10.1136/sextrans-2012-050601c.115

S Kegg, * S Goddard, J Russell. South London Healthcare NHS Trust

Background/Aims Our HIV cohort more than doubled between $2004 / 11$ with no increase in clinic capacity or staffing. Some of our service users have complex health needs-related to delayed HIV diagnosis, poor adherence to ART and co-morbidities-but an increasing number are stable on treatment and more than half have their medication delivered to their home. We are reviewing our service model to focus on the needs of our complex patients but at the same time providing a responsive, quality service to the stable patients. A key part of this review is to elicit our patients' views on the current service and how it might be improved.

Methods A self-administered questionnaire completed by $\mathrm{HIV}+$ service users.

Results 50 people completed a questionnaire and $76 \%$ described their current health as good to excellent. $43 \%$ were attending appointments 3-4 monthly, with 39\% attending more frequently. $72 \%$ wished to continue to be seen at the same frequency. Most (76\%) reported that appointments were available at suitable times. Barriers to clinic attendance included poor car-parking (44\%) and lack of transport links (13\%). A majority (84\%) have access to a personal computer and $53 \%$ have a webcam-of these $50 \%$ would value an online consultation. $32 \%$ would consider HIV monitoring by their GP and 33\% would like to receive some of their care in this setting. Of the people currently receiving ART delivered to their home $35 \%$ would like monitoring to be performed by the individual delivering the medications.

Conclusions Most patients are satisfied with the current model of care and may be reluctant to be seen less often. However there is a softening in the reluctance to involve GPs in HIV care and a willingness to consider models using newer technologies and care at home. We have reviewed our clinic template increasing the number of early and late appointments and are actively developing pilots to explore new ways to deliver care to increase choice and flexibility for our patients.

\section{P116 \\ ASSESSING THE SEXUAL VIOLENCE SERVICES CURRENTLY PROVIDED IN GENITOURINARY MEDICINE CLINICS}

doi:10.1136/sextrans-2012-050601c.116

${ }^{1} \mathrm{C}$ R Emerson, ${ }^{*} \mathrm{R}$ Sacks, ${ }^{3} \mathrm{G}$ E Foster. ${ }^{1}$ Belfast Trust; ${ }^{2}$ Imperial College Healthcare NHS Trust; ${ }^{3}$ Barts and the London

Background Many patients present to Genitourinary medicine (GUM) clinics following sexual violence (SV) occurring in circumstances including sexual assault (SA), domestic violence (DV), sex work (SW) and trafficking. BASHH has guidelines for SA but not for addressing SV in other situations. Are GUM clinics providing services for these needs?

Aims To assess services currently available in GUM for those disclosing SV.

Methods A cross sectional anonymous online survey of UK GUM clinics was performed. The survey was designed by the authors and piloted. Information was gathered on specific clinics for SV, how often they occur and which members of the multidisciplinary team are involved, and training available for SV. Links with other support services was ascertained. The data were analysed using Microsoft Excel. Results The response rate was 66/178 (37\%), with all regions and deaneries being represented. All responding clinics provide PEP, emergency contraception, STI screen, hepatitis prophylaxis and advice for those presenting after SA. $24 \%$ of clinics never use chain of evidence procedures. $80 \%$ have a local sexual assault referral centre (of these $87 \%$ were happy with this service). $80 \%, 77 \%, 50 \%$ and $38 \%$ have no dedicated clinic for female genital mutilation, sexual trafficking, DV and SW respectively and less than $50 \%$ have local training or procedures for these facets. $32 \%$ ask about nonconsensual sex in all GUM consults, 53\% ask this in special groups only. $47 \%$ routinely ask about SW.

Discussion There is a wide variation in the SV services available at GUM clinics. Sexual assault services meet guideline recommendations for GUM but SV occurring in other contexts may not be addressed. Sexual violence is increasingly reported and GUM clinics may be the first or only location of presentation. We recommend further training and guidelines to ensure clinics are equipped to meet this need.

\section{P117 AUDIT ON UPTAKE OF HIV TEST IN SEXUAL HEALTH CLINIC, ANTENATAL AND TOP SERVICES IN DUMFRIES BETWEEN JANUARY AND JUNE 2011}

doi:10.1136/sextrans-2012-050601c.117

0 Steshenko.* Dumfries and Galloway Royal Infirmary

Background BASHH introduced "UK National Guideline for HIV testing 2008" recommending universal HIV testing in GUM or sexual health clinics, antenatal and termination of pregnancy services. Early identification HIV positive persons help to reduce further transmission of HIV infection to others and allow referring them to appropriate services for further treatment.

Aims and Objectives The audit was undertaken to identify compliance of local sexual health clinic, antenatal and TOP services with "UK National Guideline for HIV testing 2008". To establish uptake of HIV test by patients accessing Sexual Health Clinic, Antenatal and TOP services in Dumfries between 1st January and 30th June 2011 and give recommendations according to findings.

Methods Retrospective audit of electronic patients' records of all persons accessing the local sexual health clinic in relation to STI issues, pregnant women attending antenatal clinic, patients receiving TOP service care between 1st January and 30th June 2011. Results From 844 episodes of care in sexual health clinic, HIV test performed in 400 (47\%) episodes. 511 women attended antenatal 